\title{
Acoso escolar y habilidades sociales en alumnado de educación básica
}

\author{
Brenda Mendoza González* y Victoria Maldonado Ramírez*
}

\begin{abstract}
Recepción: 2 de septiembre de 2015 Aceptación: 4 de mayo de 2016

*Universidad Autónoma del Estado de México, México. Correos electrónicos: brenmx@yahoo.com.mx; victoriamaldonadoramirez@gmail.com Se agradecen los comentarios de los árbitros de la revista, al DIF Municipal Atizapán de Zaragoza y a su proyecto "No permitas el bullying. Piensa Diferente" y también al apoyo económico del proyecto PRODEP DSA/103.5/14/7529.

(c) $\$ \equiv$
\end{abstract}

Resumen. Se describe la relación de habilidades sociales con el rol de participación en acoso escolar. Participaron 557 alumnos (educación primaria y secundaria). Se aplicaron dos instrumentos: competencias en habilidades sociales y bullying (índice $\alpha=0.95$ ). Los resultados describen cuatro tipos de alumnos (víctima, víctima/agresor en violencia escolar y bullying y el alumnado que no se involucra). Se observa que el alumnado que no participa en episodios de agresión tiene más habilidades sociales que los que sí se involucran. Los grupos en mayor riesgo de ser victimizados son los alumnos de primaria sin identificarse diferencias entre hombres y mujeres. Se concluye que el déficit en habilidades sociales se asocia con la participación en episodios de acoso escolar.

Palabras clave: habilidades sociales, bullying, agresión, educación básica.

\begin{abstract}
Bullying and Social Skills in Students of Basic Education

Abstract. The purpose of this study is to describe the relationship of social skills with the role of participation in school harassment. 557 students participated (primary and secondary education). Two instruments were applied: competences in social skills and bullying ( $\alpha=$ 0.95). The results describe four types of pupils (victim, victim/offender on school violence and bullying, and students who are not involved), identifying that students who do not participate in episodes of aggression, have more social skills than those who are involved, and the groups at greatest risk of being victimized are primary school pupils, without identifying differences between men and women. It is concluded that the social skills deficit is associated with participation in episodes of bullying.
\end{abstract}

Keywords: social skills, bullying, aggression, basic education.

\section{Introducción}

El bullying (acoso escolar) es un subtipo de agresión que se caracteriza por presentarse entre escolares de forma reiterada hacia un alumno en específico y se identifica desequilibrio de poder entre agresores y víctimas (Mendoza-González y Pedroza-Cabrera, 2015). Mendoza (2014a) determina que las conductas antisociales exhibidas en el aula tienen un proceso anterior, lo cual define como escalamiento en bullying. El escalamiento comienza con la exclusión entre los pares, pasan por agresión verbal y física hasta llegar a comportamientos antisociales; tiene también una amplia gama de factores de riesgo familiares, culturales, escolares e individuales que han sido organizados para un mejor estudio desde el modelo ecológico (Mendoza et al., 2014; Swearer y Shelley, 2015).
La frecuencia de hechos violentos y sus severas consecuencias en el ambiente escolar en los últimos años ha generado en México el interés por la investigación en acoso escolar. Su estudio se hace presente en la agenda del gobierno al impulsar un proyecto nacional para mejorar la convivencia en las escuelas (Plan Nacional de Desarrollo 2013-2018, 2016).

En el ámbito internacional se han desarrollado diversas investigaciones para identificar las características del alumnado que participan en ciclo de bullying en el que se desempeñan los roles de víctima, acosador, víctima-acosador, e incluso identifican factores que protegen a los alumnos que no participan en violencia escolar y bullying (Moraes y Hutz, 2010; Uribe et al., 2012; Van Hoof et al., 2008).

Recientes investigaciones identifican que uno de los factores que sitúan en mayor riesgo al alumnado para involucrarse en 
episodios de bullying es el déficit en habilidades sociales. Se observa que el alumnado que participa en bullying tiene dificultades para comunicarse asertivamente, ser empático con sus pares, iniciar y mantener conversaciones y poner en práctica diferentes formas de resolver situaciones sin hacer uso de la agresión (Cervantes, 2015; Mendoza et al., 2015; Scholte et al., 2009); entre muchas otras habilidades necesarias para interactuar de manera positiva con pares.

Las investigaciones internacionales del comportamiento de bullying y su relación con habilidades sociales se dirigen al estudio en el alumnado de nivel secundaria. En España, por ejemplo, se ha relacionado estrechamente a la empatía porque se considera modulador en el comportamiento pro-social (Mestre et al., 2002). En Chile, Nolasco (2012) determina una relación positiva y significativa entre ambas variables, y muestra que a menor nivel de empatía mayor es la participación en acoso escolar. Por su parte, Dueñas y Senra (2009) y Mendoza (2014b) proponen a la comunicación asertiva de emociones (en especial el enojo) como parte de las habilidades sociales, así como la expresión de inconformidad o situaciones conflictivas. Señalan que son factores protectores ante episodios de acoso escolar; sin embargo, en otros estudios no se han establecido relaciones entre bullying y habilidades sociales, como se describe en el estudio de Landázuri (2007), quien determina que las habilidades sociales no permiten predecir la participación del alumnado en episodios de acoso escolar como agresor o víctima en estudiantes a nivel secundaria.

En México los estudios que describen la relación del bullying con las habilidades sociales son escasos. Aún no se ha identificado la necesidad de incorporar las habilidades sociales, en especial el desarrollo de comportamiento empático en programas de intervención desarrollados por el gobierno para su implementación en recintos escolares de todos los niveles educativos (Mendoza-González y Pedroza-Cabrera, 2015). Cervantes (2015) reporta evidencia empírica a través de metodología observacional al demostrar que el alumnado de secundaria que representa el rol de víctima no es efectivo al iniciar y tampoco a mantener interacciones sociales con sus pares, siendo los que menos preferencias reciben por parte de sus pares para interactuar. La situación ha sido descrita recientemente por un grupo de especialistas canadienses, quienes determinan a través de un estudio longitudinal en estudiantes de primaria y secundaria que el alumnado víctima de bullying tiene escaso apoyo social por pares, padres y docentes. Se identifican pobres relaciones sociales con pares, con escaso apoyo y comunicación de sus padres y maestros y se describen al mismo tiempo con autopercepción pobre, en especial al autodenominarse con escasas competencias sociales, sin aceptación social de sus pares e identificando su apariencia física como negativa, así como déficit en su comportamiento (Mishna et al., 2016), es decir, como condición que los sitúa en mayor riesgo de ser elegidos como blanco de agresiones al tener una red social nula. Por lo anterior, el objetivo de este artículo es describir la relación de habilidades sociales con episodios de acoso escolar en función de los roles identificados.

\section{Método}

\section{1. Sujetos}

Participaron 557 alumnos (185 mujeres, 272 varones). El rango de edad fue entre 8 y 16 años $(M=11, \sigma=1.783)$ inscritos en seis escuelas públicas del Estado de México. Los padres de los alumnos participantes proporcionaron su autorización.

\section{2. Criterios de inclusión a la muestra}

Las escuelas fueron elegidas por tener quejas de acoso escolar en el DIF Municipal Atizapán de Zaragoza (Estado de México). En especial se eligieron aulas que manifestaron episodios de bullying.

\section{2. 1. Instrumentos utilizados}

En esta investigación se emplearon dos instrumentos: el primero de ellos fue el de Actitudes y Estrategias Cognitivas Sociales (AECS) (Moraleda et al., 1998), que evalúa la competencia social (facilidad o dificultad del alumnado para adaptarse a su contexto social). Para el proyecto se hizo la validación para población mexicana. Su índice de confiabilidad en estudiantes mexicanos es de $\alpha=0.95$. Para medir el proceso de socialización, la prueba se conforma de 137 reactivos divididos en dos factores:

a) Escala de actitud social (conformidad con lo socialmente correcto, sensibilidad social o empatía, ayuda y colaboración, seguridad y firmeza en la interacción, liderazgo prosocial, agresividad, dominancia, apatía y retraimiento, ansiedad, timidez).

b) Escala de pensamiento social (impulsividad, independencia, convergencia, percepción y expectativas sobre la relación social, percepción acerca de liderazgo de sus padres, aceptación por parte de sus padres, observación de situaciones sociales, solución de problemas sociales, consecuencias al comportamiento social y elección de la alternativa más adecuada). Se aplica de forma individual y colectiva.

El segundo instrumento usado fue el Cuestionario de Evaluación de la Violencia y Acoso Escolar que ha sido validado para alumnado en nivel primaria y secundaria en estudiantes mexicanos (Mendoza-González et al., 2015). Consta de 51 reactivos que permiten identificar la participación del alumno en episodios de acoso escolar como víctima, acosador 
o testigo. Tiene cuatro opciones de respuesta (todos los días, dos o tres veces por semana, dos o tres veces al mes y no ha sucedido). Índice de confiabilidad general de $\alpha=0.95$. A continuación se describen los factores:

a) Factor rol víctima: comportamientos de agresión y exclusión que recibe de otros pares (17 reactivos, i. e., me ignoran, me impiden participar, me roban cosas, me amenazan con armas). El participante informa la frecuencia con la que suceden los episodios.

b) Factor rol agresor: comportamiento de agresión y exclusión en la que el alumno participa ejerciendo agresión hacia otros; está conformado por 17 reactivos (yo ignoro, yo impido que otros participen, yo robo cosas, yo amenazo con armas).

c) Factor rol observador: evalúa el rol de observador cuando identifica conductas agresivas que otros exhiben hacia sus pares; está conformado por 17 reactivos (he observado que ignoran, he observado que un compañero impide que otro participe, he observado que roban cosas, he observado que amenazan).

\section{Procedimiento}

Esta investigación se deriva de un macroproyecto financiado por Prodep (SEP) y coordinado por Facultad de Ciencias de la Conducta y el Sistema Municipal DIF Atizapán de Zaragoza (2013-2015).

Los centros escolares fueron elegidos conforme a las quejas de episodios de bullying recibidas en el DIF Atizapán de Zaragoza. La autorización para que participaran los centros educativos y el alumnado fue gestionada por la misma institución. Los instrumentos se aplicaron en dos sesiones, en aulas escolares con ventilación e iluminación adecuada. La captura y análisis de los datos se elaboró con el programa estadístico sPss versión 16. Se realizó una prueba de bondad de ajuste (prueba de Kolmogórov-Smirnov) con el objetivo de probar o no la hipótesis nula. Se identifica que la muestra es con distribución normal $(p>0.05)$, por lo que se justificó el uso de pruebas paramétricas.

\section{Resultados}

\section{1. Tipología en los estudiantes participantes}

Para conocer la relación entre las dos variables del estudio (bullying y habilidades sociales), se identificaron los roles de los alumnos que participan en episodios de bullying. Para obtener la clasificación se hizo un análisis $K$ de medias que combina los factores agresor y víctima del instrumento de agresión en la escuela con base en los siguientes factores: a) victimización extrema, b) victimización media, $c$ ) victimización excluyente, $d$ ) agresión extrema, $e$ ) agresión media y f) agresión excluyente.

En el cuadro 1 se muestran las medias de los conglomerados para cada una de las situaciones en las que los alumnos participan en episodios de acoso escolar en interacción con sus compañeros.

\section{1. 1. Grupo 1. Víctima/agresor en violencia escolar}

Este grupo está constituido por 26 alumnos, de los cuales 9 son mujeres que representan $2 \%$ de todos los alumnos y 17 varones que reflejan 3\% de la población total participante. Son los alumnos que ejercen agresión media a través de insultos, apodos, golpes, empujones, burlas y esconden pertenencias de sus compañeros y son víctimas al mismo tiempo. Son alumnos que reciben y ejercen comportamiento de exclusión entres sus pares: rechazar, ignorar, prohibir participar y hablar mal de otros. Los episodios de agresión ocurren una o dos veces por mes.

\section{1. 2. Grupo 2. Víctima de exclusión de bullying}

Se conforma por 33 alumnos que reciben agresión excluyente cotidiana por medio de apodos, burlas, son rechazados, les prohíben participar y hablan mal de ellos, de los cuales 12 son mujeres que representan $2 \%$ del total de alumnos y 21 varones que conforman $4 \%$ de la población.

\section{1. 3. Grupo 3. Victima/agresor de bullying}

Son los alumnos que ejercen agresión extrema (amenazan con armas, amenazan para intimidar, amenazan con insultos de carácter sexual, con armas, mensajes por internet o roban), agresión media (empujar, golpear, insultos, apodos, esconden o rompen pertenencias), exclusión (rechazo, ignorar, impiden participar, hablan a espaldas de sus compañeros). Ejercen agresión media y al mismo tiempo son víctimas de ello.

El grupo está conformado por 9 participantes, 2 son mujeres que representan $0.3 \%$ del total de participantes y 7 hombres que representan 1\% del total de participantes. Los episodios de agresión ocurren cotidianamente.

\begin{tabular}{|c|c|c|c|c|}
\hline Grupo & 1 & 2 & 3 & 4 \\
\hline Víctima de agresión extrema & 11.81 & 11.7 & 19.89 & 7.29 \\
\hline Víctima de agresión media & 8.44 & 10.06 & 12.78 & 5.17 \\
\hline Víctima de exclusión & 14.8 & 24.24 & 26.56 & 10.03 \\
\hline Agresión extrema & 14.57 & 9.52 & 27.11 & 9.15 \\
\hline Agresión media & 14.47 & 8.24 & 20.67 & 6.89 \\
\hline Agresor de exclusión & 8.15 & 6.48 & 12.33 & 4.70 \\
\hline
\end{tabular}




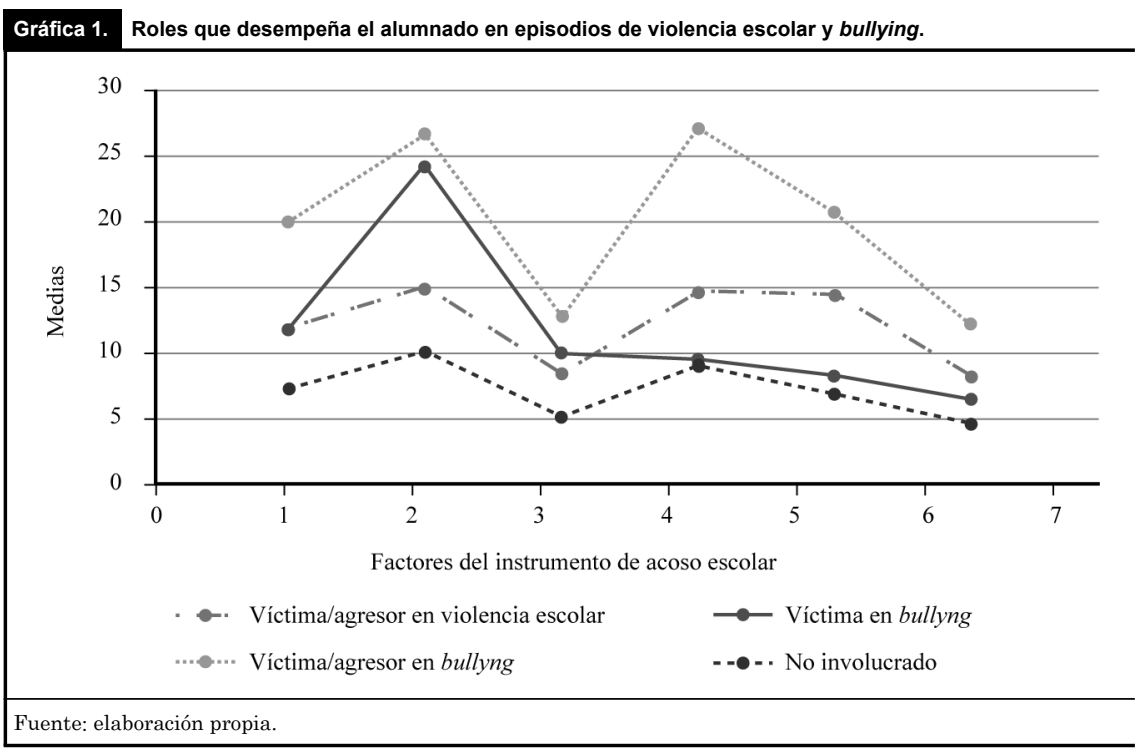

Cuadro 2.

Factores de la Escala de actitud social con diferencias significativas de medias.

\begin{tabular}{|c|c|c|c|c|c|c|c|c|}
\hline & \multicolumn{2}{|c|}{$\begin{array}{l}\text { Víctima/agresor } \\
\text { (violencia escolar) }\end{array}$} & \multicolumn{2}{|c|}{$\begin{array}{l}\text { Víctima de } \\
\text { bullying }\end{array}$} & \multicolumn{2}{|c|}{$\begin{array}{l}\text { Víctima /agresor } \\
\text { de bullying }\end{array}$} & \multicolumn{2}{|c|}{ No involucrado } \\
\hline & $\bar{x}$ & $\sigma$ & $\bar{x}$ & $\sigma$ & $\bar{x}$ & $\sigma$ & $\bar{x}$ & $\sigma$ \\
\hline Sensibilidad social (SEN) & 35.7 & 13.75 & 39.41 & 11.10 & 26.87 & 12.74 & 42.28 & 10.89 \\
\hline Ayuda y colaboración (AC) & 41.66 & 13.87 & 47.04 & 13.49 & 29.42 & 9.58 & 50.35 & 12.96 \\
\hline Ansiedad-timidez (ANS) & 25.56 & 9.46 & 28.87 & 8.17 & 29.28 & 13.03 & 23.25 & 9.28 \\
\hline Apatía-retraimiento (AP) & 34.64 & 10.37 & 34.53 & 6.73 & 35.16 & 11.54 & 29.8 & 9.78 \\
\hline
\end{tabular}

Cuadro 3.

Factores de la Escala de pensamiento social con diferencias significativas de medias.

\begin{tabular}{|c|c|c|c|c|c|c|c|c|}
\hline & \multicolumn{2}{|c|}{$\begin{array}{l}\text { Víctima/agresor } \\
\text { (violencia escolar) }\end{array}$} & \multicolumn{2}{|c|}{$\begin{array}{l}\text { Víctima de } \\
\text { bullying }\end{array}$} & \multicolumn{2}{|c|}{$\begin{array}{l}\text { Víctima /agresor } \\
\text { de bullying }\end{array}$} & \multicolumn{2}{|c|}{ No involucrado } \\
\hline & $\bar{x}$ & $\sigma$ & $\bar{x}$ & $\sigma$ & $\bar{x}$ & $\sigma$ & $\bar{x}$ & $\Sigma$ \\
\hline $\begin{array}{l}\text { Impulsividad frente a la } \\
\text { reflexividad (IMP) }\end{array}$ & 29.72 & 9.87 & 28.24 & 8.33 & 27.37 & 7.9 & 22.45 & 9.85 \\
\hline $\begin{array}{l}\text { Percepción y expectativas } \\
\text { sobre la relación social (PER) }\end{array}$ & 21.4 & 7.19 & 24.73 & 8.74 & 23 & 9.48 & 18.39 & 8.68 \\
\hline $\begin{array}{l}\text { Habilidad en la observación } \\
\text { y retención de la informa- } \\
\text { ción relevante sobre las si- } \\
\text { tuaciones sociales (OBS) }\end{array}$ & 30.08 & 10.5 & 25.89 & 10.45 & 25 & 13.14 & 22.23 & 10.39 \\
\hline $\begin{array}{l}\text { Habilidad para anticipar y } \\
\text { comprender las consecuen- } \\
\text { cias que posiblemente se } \\
\text { seguirán de los comporta- } \\
\text { mientos sociales (CONS) }\end{array}$ & 27.04 & 10.03 & 24.17 & 9.05 & 25 & 14.43 & 21.03 & 10.33 \\
\hline $\begin{array}{l}\text { Habilidad en la búsqueda } \\
\text { de soluciones alternativas } \\
\text { para resolver los problemas } \\
\text { sociales (ALT) }\end{array}$ & 27.95 & 10.54 & 28.32 & 9.52 & 29.75 & 12.03 & 24.07 & 9.93 \\
\hline $\begin{array}{l}\text { Habilidad para elegir los } \\
\text { medios adecuados a los fines } \\
\text { que se persiguen en el com- } \\
\text { portamiento social (MED) }\end{array}$ & 28.71 & 10.64 & 28.2 & 10.16 & 29.71 & 12.41 & 22.82 & 10.73 \\
\hline
\end{tabular}

\section{1. 4. Grupo 4. No involucrado}

El grupo lo conforman 489 estudiantes que no participan en situaciones de violencia escolar o bullying. De los varones, 240 representan $43 \%$ de los participantes y 249 son mujeres que conforman $44.7 \%$ de la población total.

En la gráfica 1 se representan los roles o tipos de estudiantes identificados: Víctima/agresor en violencia escolar (grupo 1), Víctima de bullying (grupo 2), Víctima/agresor de bullying (grupo 3) y No involucrado (grupo 4) en el total de los participantes.

\section{2. Rol que desempeña el alum- nado en situaciones de bullying y las habilidades sociales que exhibe.}

Se realizó la prueba ANOva y se encontraron las diferencias significativas que existen entre el rol que desempeña el alumno en episodios de bullying y los factores correspondientes a la escala de actitud social y pensamiento social (cuadro 2, cuadro 3). Al respecto, se presentan las medias estadísticamente significativas.

A continuación se describen los análisis de varianza realizados para cada uno de los factores de la Escala de actitud social del instrumento (cuadro 2).

El análisis de varianza realizado permitió identificar que en la Escala de actitud social, el factor de sensibilidad social presenta diferencias de medias significativas $F(d f 1=3$ y $d f 2=510)=$ $2.576, p<0.001$. El grupo 3 Víctima/ acosador de bullying tiene media menor que el grupo 4 No involucrado y que el grupo 2 Víctima de bullying.

Para el factor de ayuda y colaboración existen diferencias de medias significativas $F(d f 1=3$ y $d f 2=505)=$ $7.915, p<0.001$. El grupo 4 No involucrado tiene media significativamente más alta que el alumnado de grupo 1 Víctima/agresor en violencia escolar y que el grupo 3 Víctima/acosador de 
bullying. Este último tiene media más baja que el grupo 2 Víctima de bullying.

En el factor de ansiedad-timidez se encontraron diferencias significativas de medias $F(d f 1=3$ y $d f 2=503)=4.687, p<$ 0.05. El grupo 2 Víctima de bullying tiene media significativamente mayor en este factor que el grupo 4 No involucrado.

En apatía-retraimiento se encontraron diferencias significativas de medias $F(d f 1=3$ y $d f 2=498)=4.339, p<0.05$. El grupo 2 Víctima de bullying y el grupo 1 Víctima/acosador de violencia escolar presentan media significativamente mayor que el grupo 4 No involucrado.

En el cuadro 3 se describen los análisis de varianza realizados para cada uno de los factores de la Escala de pensamiento social del instrumento. En las habilidades para esta escala y debido a la estructura dentro del instrumento se establece que las puntuaciones bajas refieren la competencia de manera positiva y las puntuaciones altas deliberan una exhibición negativa de la habilidad.

El factor de impulsividad frente a la reflexión presenta diferencias de medias significativas $F(d f 1=3$ y $d f 2=512)=7.240$, $p<0.001$. Los alumnos del grupo 4 No involucrado tienen media significativamente menor que el grupo 1 Víctima/ agresor en violencia escolar y que el grupo 2 Víctima de bullying.

En el factor de percepción y expectativas sobre la relación social existen diferencias significativas de medias $F(d f 1=3 \mathrm{y}$ $d f 2=522)=6.295, p<0.001$. El grupo 2 Víctima de bullying exhibe un nivel más alto en esta habilidad que el grupo 4 No involucrado.

En la observación y retención de la información relevante sobre la situaciones sociales también se encontraron diferencias significativas de medias $F(d f 1=3$ y $d f 2=515)=5.141$, $p<0.05$. El grupo 1 Víctima/agresor en violencia escolar exhibe un déficit en esta habilidad a diferencia del grupo 4 No involucrado.

En la habilidad para participar y comprender las consecuencias de las acciones de sus comportamientos $F(d f 1=3$ y $d f 2=510)=3.696, p<0.05$. El grupo 1 Víctima/agresor en violencia escolar presenta una puntuación más alta que el grupo 4 No involucrado.

Para la habilidad en la búsqueda de soluciones alternativas para resolver los problemas sociales $F(d f 1=3$ y $d f 2=502)$ $=3.290, p<0.05$, las pruebas post hoc identifican diferencias significativas. El grupo 2 Víctima de bullying presenta un déficit en esta habilidad en comparación con el grupo 4 No involucrado.

En la habilidad para elegir los medios adecuados a los fines que se persiguen en el comportamiento social $F(d f 1=3$ y $d f 2=508)=4.901, p<0.05$. La prueba post hoc indica que hay diferencias. El grupo 4 No involucrado presenta mayor destreza en la elección de los medios para resolver problemas que el grupo 1 Víctima/agresor en violencia escolar y que el grupo 2 Víctima de bullying.

\section{3. Diferencias de género y escolaridad}

\section{3. 1. Género}

Para identificar una dependencia entre el rol que desempeña el alumnado en violencia o acoso escolar, se realizó $\chi^{2}$ y los resultados indican que son independientes: $\chi^{2}=(3 \mathrm{gl})=7.560^{\mathrm{a}}$ $\operatorname{con} p=0.056$.

\section{3. 2. Escolaridad}

Para tener conocimiento de la dependencia entre variables de escolaridad (primaria y secundaria) y los roles identificados en violencia escolar y bullying, se realizó $\chi^{2}$. Los resultados indican que son dependientes $\left[\chi^{2}=(3 \mathrm{gl})=10.293^{\mathrm{a}} \operatorname{con} p<0.05\right]$.

A continuación en el cuadro 4 se presentan los residuos corregidos de la tipología identificada que se realizaron para determinar el grupo que presenta un mayor riesgo en función de la escolaridad con $V$ de Cramer $=0.136, p<0.05$.

Los resultados de los residuos corregidos en el cuadro 4 indican que el alumnado de primaria se encuentra en mayor riesgo que el alumnado de secundaria de participar en episodios de bullying en el rol de víctima.

\section{Discusión}

Los resultados de este estudio son pioneros al demostrar los roles de participación del alumnado en episodios de bullying. En México aún no se ofrece evidencia empírica sobre los roles de participación en estudios nacionales. Desde hace varios años la investigación internacional ofrece el análisis de los roles de participación como punto de partida para cualquier tipo de estudio con línea en acoso escolar. Se establece que los roles que desempeña el alumnado en episodios de violencia y acoso escolar son los de víctima/agresor en violencia escolar, víctima pura en bullying, víctima/agresor en bullying. La gran mayoría del alumnado no participa en episodios

\begin{tabular}{|c|c|c|c|c|}
\hline & $\begin{array}{l}\text { Víctima/agresor } \\
\text { (violencia escolar) }\end{array}$ & $\begin{array}{c}\text { Víctima de } \\
\text { bullying }\end{array}$ & $\begin{array}{c}\text { Víctima /agresor de } \\
\text { bullying }\end{array}$ & $\begin{array}{c}\text { No } \\
\text { involucrado }\end{array}$ \\
\hline Género & \multicolumn{4}{|c|}{ Residuo corregido } \\
\hline Primaria & -1.7 & 2.3 & 1.6 & -1.2 \\
\hline Secundaria & 1.7 & -2.3 & -1.6 & 1.2 \\
\hline
\end{tabular}


de bullying y representa el rol No involucrado (Cerezo y Ato, 2010; Moraes y Hutz, 2010; Terrazo et. al., 2011). Las primeras investigaciones en los años setenta realizadas por Olweus (2006) identificaban tres roles: agresor, víctima y espectador. Otras investigaciones posteriores coincidían con ello (Correia y Dalbert, 2008; Dijkstra et al., 2008, Horne et al., 2007, Jones et al., 2008). Recientemente la revisión y análisis de la evidencia experimental ha permitido identificar a cuatro roles básicos desempeñados por el alumnado en episodios de bullying: víctima, espectador, acosador y el doble rol víctima/ acosador (Magklara et al., 2012). Este último es uno de los aportes significativos de la investigación en la línea del bullying.

El alumnado con doble rol (víctima/agresor) frecuentemente genera confusión en las autoridades escolares y representa un reto en su estudio para los investigadores. Existe amplia evidencia que describe a este alumnado (Moraes y Hutz, 2010), incluso se han dedicado investigaciones particularmente a su estudio que definen diferencias con los otros roles (Mendoza et al., 2015; Terrazo et al., 2011), por lo que los resultados de este estudio aportan evidencia que fortalece a la línea de investigación de bullying especialmente en las características del alumnado que desempeña cada rol en aulas mexicanas.

La evidencia encontrada permitió identificar diferencias entre la víctima, la víctima/acosador y el alumnado que no se involucra. El alumnado victimizado es más ansioso y tímido, que son variables que interfieren en las habilidades necesarias para afrontar situaciones de agresión con sus pares (Moraes y Hutz, 2010; Ok y Aslan, 2010; Tomsa et al., 2013), lo que confirma la evidencia en otras investigaciones (Cava, 2011; Cava et al., 2010; Cerezo y Ato, 2010; Cervantes, 2015; Polo del Río et al., 2013). Los sitúa incluso en mayor riesgo de suicidio y depresión (Ceccatelli et al., 2010; Hunter et al., 2007). Son más apáticos a la interacción con sus pares y retraídos (le disgusta integrarse a grupos y actividades por equipo, le gusta "estar solo"). Este hecho se explica con la dificultad que tienen para iniciar y mantener una conversación con sus pares y relacionarse con ellos, ya que por lo general no se dirigen de una manera exitosa hacia sus compañeros lo que se asocia con su exclusión constante de los grupos sociales y al mismo tiempo son elegidos para ser agredidos. El déficit en habilidades sociales evita que la víctima tenga la capacidad de afrontar las situaciones conflictivas y responda de manera complementaria a las acciones del agresor provocando que la conducta agresiva se mantenga (Cervantes, 2015).

Derivado de los resultados encontrados, que al mismo tiempo fortalecen a los otros, se identifica que el alumno víctima de bullying tiene un déficit en habilidades para generar, evaluar y elegir alternativas de solución adecuadas ante situaciones problemáticas en las que interviene la creatividad para afrontarlas (Avilés, 2009; Azebedo et al., 2012). Estos resultados refieren a lo que se determina la distorsión cognitiva de pensamiento ilusorio conocida también como wishful thinking; significa que el alumnado crea historias sin sustento real, exhibe en ellas sus deseos y no lo que le ocurre en su vida cotidiana, hace creer que nada malo sucede, por lo que no hay que solucionar ninguna situación problemática. Por tal motivo la evidencia encontrada fortalece esta distorsión cognitiva, ya se demuestra que el niño víctima de bullying carece de estrategias para solucionar conflictos y utiliza el pensamiento ilusorio (wishful thinking) como estrategia de evasión a la situación problemática (Hunter et al., 2007).

Respecto al alumnado que desempeña el doble rol víctima/ acosador, se identificó que son los alumnos menos empáticos los que se encuentran menos dispuestos a ayudar y cooperar con sus pares. Los resultados contrastan con las características del alumnado no involucrado que no participa en situaciones de violencia y acoso escolar, es más empático, tiene mayor control de sus emociones (menos impulsivos), valora las situaciones problemáticas de forma objetiva, busca alternativas de solución sin recurrir a la agresión y se hace responsable de sus actos, por lo que identifican su comportamiento agresivo sin justificarlo. Mestre et al. (2002) y Mendoza (2014a) sostienen que la falta de control sobre las emociones y la impulsividad son detonantes para el desarrollo de la agresividad. Además, reconocen que el autocontrol es un factor que protege al alumnado de involucrarse en episodios de violencia y acoso escolar. Son los alumnos más empáticos (sensibilidad social) en la relación que establecen con pares. Stan y Galea (2014) señalan que la empatía se relaciona con un autoconcepto positivo y permite establecer con facilidad relaciones con los demás al facilitar el afrontamiento de situaciones conflictivas.

Este tipo de alumno que no se involucra en episodios de acoso escolar demostró que su percepción y expectativas de las relaciones sociales que establecen con sus pares son positivas: expresan asertivamente sentimientos positivos y negativos, defienden derechos y formas de pensar, hacen peticiones y los rechazan de manera asertiva. Los resultados fortalecen lo señalado por otros investigadores (Eceiza et al., 2008). Se reconoció también que posee la habilidad de observar y retener información relevante de situaciones sociales, lo que le permite responder y participar constantemente con sus pares, presenta mayores habilidades para encontrar soluciones alternativas en problemas sociales reflexionando y analizando antes de actuar. Son alumnos que ayudan, cooperan con sus pares y se confirma lo observado en estudiantes de otros niveles educativos como bachiller: el alumnado que no se involucra en episodios de bullying responde de manera asertiva ante las agresiones de sus 
compañeros. El alumnado ayuda a través de redes sociales, coopera con iguales y recibe también ayuda de sus amigos, lo que se denomina reciprocidad (Mendoza et al., 2015).

Otro objetivo fue conocer la diferencia de género respecto al comportamiento de bullying. Al respecto, hombres y mujeres se encuentran en igual riesgo de ejercer o recibir agresión de sus compañeros, lo cual es congruente con otros estudios (Azebedo et al., 2012; Moraes y Hutz, 2010), cuya explicación puede basarse en los niveles de violencia en la sociedad mexicana y que se reflejan en los contextos escolares (INEGI, 2015).

Las diferencias del acoso escolar respecto al nivel educativo muestra que sí existen diferencias significativas entre estudiantes de primaria y secundaria, datos que no son consistentes con lo reportado por Sánchez y Cerezo (2010), quienes determinan que el nivel educativo no es un factor determinante en España en la incidencia del bullying y generaliza el fenómeno tanto en primaria como en secundaria; sin embargo, las conclusiones de este artículo determinan que es un factor dependiente, ya que en primaria el alumnado se encuentran en mayor riesgo de desempeñar el rol de víctima que el alumnado de secundaria. Estos resultados fortalecen otros reportes de investigación (Muñoz, 2008).

Es importante reconocer que una limitante del estudio fue que se desarrolló en un único municipio del Estado de México; por lo tanto, no se deben generalizar los resultados. A pesar de lo anterior, hay un aporte de evidencia que permite tener una guía para el diseño de programas de prevención e intervención en niveles individuales para los diferentes roles de alumnos que participan en episodios de bullying.

\section{Prospectiva}

Gutiérrez et al. (2011) establecen que las relaciones interpersonales son aprendidas con base en la interacción con los demás y determinan las habilidades sociales como un factor indispensable para afrontar, resolver y tomar decisiones ante situaciones conflictivas como ejes en las relaciones sociales.

De este modo, los resultados de este estudio apoyan la evidencia de la creación de programas que faciliten el aprendizaje de habilidades para convivir positivamente con pares, como elementos protectores ante episodios de agresión con el objetivo de mejorar la convivencia y fortalecer la autonomía en el alumnado (González-Arratia y Valdez, 2013; Siquirdson et al., 2014). La evidencia empírica ha demostrado su pertinencia describiéndose algunos componentes en los programas de convivencia escolar (Mendoza-González y Pedroza-Cabrera, 2015):

a) Relajación (dirigido a disminuir la ansiedad y aumentar autocontrol)

b) Habilidades sociales (fortalecer redes sociales entre pares)

c) Comunicación asertiva (incrementar la participación en actividades sociales)

d) Aprendizaje cooperativo (fortalecer el trabajo en equipo)

e) Autocontrol (disminuir impulsividad)

f) Solución de problemas (incrementar creatividad y análisis la solución de problemas)

Por los motivos expuestos se sugiere implementarlos en programas para mejorar la convivencia entre estudiantes.

Referencias

Avilés, J. M. (2009). Victimización percibida y bullying. Factores diferenciales entre víctimas. Boletin de Psicología, 95, 7-28.

Azebedo, R., Vanila, R., Azebedo, T., Sica, A. L., Jansen, K., Lessa, B., Dias de Mattos, L. y Tavares, R. (2012). Bullying and associated factors in adolescents aged 11 to 15 years. Trends in Psycbiatry and Psychotherapy, 34(1), 19-24.

Cava, M. J. (2011). Familia, profesorado e iguales: claves para el apoyo a las víctimas de acoso escolar. Psychosocial Intervention, 20(2), 183-192.

Cava, M. J., Musitu, G., Buelga, S. y Murgui, S. (2010). The relationships of family and classroom environments with peer rela- tional victimization: An analyisis of their gender differences. The Spanish Journal of Psychology, 13 156-165.

Ceccatelli, C., Marianacci, R. y Cateo, A. (2010). Lisrel model for a confirmatory analysis: relationship between low selfworth level and victim of bullying. Procedia Social and Behavior Siences, 30, 628-644.

Cerezo, F. y Ato, M. (2010). Social status, gender, classroom climate and bullying among adolescents pupils. Anales de Psicología, 26(1), 137-144.

Cervantes, A. R. (2015). Procesos de interacción social en adolescentes bullying (tesis doctoral). Aguascalientes: Universidad Autónoma de
Aguascalientes.

Correia, I. y Dalbert, C. (2008). School bullying belief in a personal just world of bullies, victims, and defenders. European Psychologist, 13(4), 248-254

Dijkstra, J., Lindenberg, S. y Veenstra, R. (2008). Beyond the class norm: bullying behavior of popular adolescents and its relation to peer acceptance and rejection. Journal of Abnormal Child Psychology, 36, 1289-1299.

Dueñas, M. L. y Senra, M. (2009). Habilidades sociales y acoso escolar: un estudio en centros de enseñanza secundaria en Madrid. Revista Española de Orientación y Psicopedagogía, 20(1), 39-49. 
Eceiza, M., Arrieta, M. y Goñi, A. (2008). Habilidades sociales y contextos de la conducta social. Revista de Psicodidáctica, 13(1), 11-26.

González-Arratia, N. I. y Valdez, J. L. (2013). Optimismo-pesimismo y resiliencia en adolescentes de una universidad pública. CIENCIA ergo-sum, 19(3), 207-214.

Gutiérrez, M., Escartí, A. y Pascual, C. (2011). Relaciones entre empatía, conducta prosocial, agresividad, autoeficacia y responsabilidad personal social de los escolares. Psicothema, 23(1), 13-19.

Horne, A., Stoddard, J. y Bell, C. (2007). Group approaches to reducing aggression and bullying in school. Group Dynamics: Theory, Research, and Practice, 11(4), 262-271.

Hunter, S. C., Boyle, J. y Warden, D. (2007) Perceptions and correlates of peervictimization and bullying. British Journal of Educational Psychology, 77(4), 797-810.

INEGI (Instituto Nacional de Estadística y Geografía) (2015). Encuesta Nacional de Seguridad Pública Urbana (ENSU). Aguascalientes.

Jones, S., Haslam, S. A., York, L. y Ryan, M. (2008). Rotten apple or rotten barrel? Social identity and children's responses to bullying. British Journal of Developmental Psychology, 26, 117-132.

Landázuri, V. (2007) Asociación entre el rol de agresor y el rol de víctima de intimidación escolar, con la autoestima y las habilidades sociales de adolescentes de un colegio particular mixto de lima. Revista Psicológica Herediana, 2(2), 71-80.

Magklara, K., Skapinakis, P., Gkatsa, T., Bellos, S., Araya, R., Stylianidis, S. y Mavreas, V. (2012). Bullying behaviour in schools, socioeconomic position and psychiatric morbidity: a crosssectional study in late adolescents in Greece. Child and Adolescent Psychiatry and Mental Health, 6(8), 1-13.

Mendoza, B. (2014a). Bullying: los múltiples rostros del acoso escolar (2a ed). México: Pax México.

Mendoza, B. (2014b). Asambleas escolares. Estrategia para resolver conflictos a través de competencias. México: Pax México.

Mendoza, B., Morales, T. y Arriaga, Y. (2015). Variables proximales relacionados con violencia escolar y bullying en alumnado de bachillerato. Special Issue of Psychology, Society and Education Journal, 7(2), 185-200.

Mendoza, B., Pedroza, F. y Martinez, K. (2014). Prácticas de crianza positiva: entrenamiento a padres para reducir el bullying. Acta de Investigación Psicológica, 4(3), 1793-1808.

Mendoza-González, B., Cervantes-Herrera, A. R., Pedroza-Cabrera, F. J., AguileraRubalcava, S. J. (2015). Estructura factorial y consistencia interna del cuestionario para medir bullying y violencia escolar. Revista Ciencia UAT, 10(1), 6-16.

Mendoza-González, B. y Pedroza-Cabrera, F. J. (2015). Evaluación de un programa de intervención para disminuir el acoso escolar y la conducta disruptiva. Acta de Investigación Psicológica, 5(2), 1947-1959.

Mestre, M. V., Samper, P. y Frías, M. D. (2002). Procesos cognitivos y emocionales predictores de la conducta prosocial y agresiva: la empatía como factor modulador. Psicothema, 14(2), 227-232.

Mishna, F., Khoury-Kassabri, M., Schwan, K., Wiener, J., Craig, W., Beran, T., Pepler, D. y Daciuk, J. (2016). The contribution of social support to children and adolescents' self-perception: The mediating role of bullying victimization. Children and Youth Services Review, 63,120-127.

Moraes, C. de y Hutz, C. (2010). As implicações do bullying na autoestima de adolescentes. Psicologia Escolar e Educacional, 14(1), 131-138.

Moraleda, M., González Galán, A. y GarcíaGallo, J. (1998) Actitudes y estrategias cognitivas sociales (AECS). Madrid: TEA Ediciones.

Muñoz, G. (2008). Violencia escolar en México y en otros países: comparaciones a partir de los resultados del Instituto Nacional para la Evaluación de la Educación. Revista Mexicana de Investigación Educativa, 13(39), 1195-1228.

Nolasco, A. (2012). La empatía y su relación con el acoso escolar. Revista de Estudios y Experiencias en Educación, 11(22), 35-54.

Ok, S. y Aslan, S. (2010). The school bullying and perceived parental style in adolescents. Procedia Social and Behavioral Sciences, 5 , 536-540.

Olweus, D. (2006). Una revisión general, en A. Serrano (ed.). Acoso y violencia en la escuela. ¿Cómo detectar, prevenir y resolver el bullying? (pp. 79-106). Barcelona: Ariel.

Plan Nacional de Desarrollo 2013-2018 (2016).
Disponible en http://pnd.gob.mx/. Consultado el 25 de enero de 2016.

Polo del Río, M. I., León del Barco, B., Gómez, T., Palacios, V. y Fajardo, F. (2013). Estilos de socialización en víctimas de acoso escolar. European Journal of Investigation in Health, Psychology and Education, 3(1), 41-49.

Sánchez, C. y Cerezo, F. (2010). Variables personales y sociales relacionadas con la dinámica bullying en escolares de educación primaria. Electronic Journal of Researchin Educational Psychology, 8(2), 1015-1032.

Scholte, R., Overbeek, G., Brinkten, G., Rommes, E., Kemp, R., Goossens, L., Engels, R. (2009). The significance of reciprocal and unilateral frienships for peer victimization in adolescence. Journal of Youth Adolescence, 38, 89-100.

Siquirdson, J. F., Wallander, J. y Sund, A. N. (2014). Is involvement in school bullying associated with general health and psychosocial adjustment outers in adulthood? Child abuse and neglect, 38, 1607-1617.

Stan, C. y Galea, I. (2014). The development of social and emotional skills of student's ways to reduce the frequency of bullying-type events. Experimental results. Procedia-Social and Behavioral Sciences, 114, 735-743.

Swearer, S. y Shelley, H. (2015). Understanding the psychology of bullying: Moving toward a social-ecological diathesis-stress model. American Psychologist, 70(4), 344-353.

Terrazo, M., Ossorno, S., Babarro, J. M. de y Martínez, R. (2011) Social characteristics in bullying typology: Digging deeper into description of bully- victim. Procedia-Social and Behavioral Sciences, 29, 869-878.

Tomsa, R., Jenaro, C., Campbell, M. y Neacsu, D. (2013). Student's experiences with tradicional bullying and cyberbullying: findings from a Romanian simple. Social and Behavioral Sciences, 78, 586-590.

Uribe, A. F., Orcasita, L., Aguillón, E. (2012). Bullying, redes de apoyo social y funcionamiento familiar en adolescentes de una institución educativa de Santander. Avances de la Disciplina, 6(2), 83-99.

Van Hoof, A., Raaijmakers, Q., Van Beek, Y., Hale, W. y Aleva, L. (2008). A multi-mediation model on the relations of bullying, victimization, identity, and family with adolescent depressive symptoms. Journal Youth Adolescence, 37, 772-782. 